\title{
Update on the Management of Cholangiocarcinoma
}

\author{
J.R.A.Skipworth ${ }^{a}$ M.G. Keane ${ }^{b}$ S.P. Pereira ${ }^{b}$ \\ ${ }^{a}$ Department of Surgery and Interventional Science, University College London, and ${ }^{\mathrm{b}}$ Institute for Liver and \\ Digestive Health, University College London Medical School, London, UK
}

\section{Key Words}

Biliary tract carcinoma $\cdot$ Cholangiocarcinoma . Surgery .

Neoadjuvant therapy · Adjuvant therapy

\begin{abstract}
Cholangiocarcinoma (CC) is a rare cancer arising from the epithelium of the biliary tree, anywhere from the small peripheral hepatic ducts to the distal common bile duct. Classification systems for CC typically group tumours by anatomical location into intrahepatic, hilar or extrahepatic subtypes. Surgical resection or liver transplantation remains the only curative therapy for CC, but up to $80 \%$ of patients present with advanced, irresectable disease. Unresectable CC remains resistant to many chemotherapeutic agents, although gemcitabine, particularly in combination with other agents, has been shown to improve overall survival. Ongoing investigation of biological agents has also yielded some promising results. Several novel interventional and endoscopic techniques for the diagnosis and management of non-operable CC have been developed: initial results show improvements in symptoms and progression-free survival, but further randomised studies are required to establish their role in the management of CC.

(c) 2014 S. Karger AG, Basel
\end{abstract}

\section{Introduction}

Cholangiocarcinoma (CC) is a rare tumour of cholangiocytes (epithelia of the biliary tract) [1] with an incidence in the UK of approximately 1-2 per 100,000 population $[2,3]$. However, the incidence (particularly of intrahepatic tumours) continues to rise annually both within the UK and worldwide [3-6], and in the mid-1990s CC overtook hepatocellular carcinoma as the commonest cause of liver cancer death in the UK [7]. Established risk factors for CC include male sex, increasing age, smoking and diabetes [4].

Up to $70 \%$ of CC arise from the hilar region (also known as Klatskin tumours) and involve the main extrahepatic bile duct and right or left hepatic ducts [1]. Almost all other tumours are extrahepatic, involving the distal common bile duct [1]. Hilar and extrahepatic tumours typically cause progressive occlusion of the external biliary tree and present with painless obstructive jaundice, with or without cholangitis. Only rarely do tumours arise from smaller intrahepatic bile ducts, often forming a solitary, compressive mass that may result in non-specific symptoms such as right upper quadrant discomfort, anorexia and nausea.

\section{KARGER}

E-Mail karger@karger.com

www.karger.com/ddi
(C) 2014 S. Karger AG, Basel

0257-2753/14/0325-0570\$39.50/0
Stephen P. Pereir

UCL Institute for Liver and Digestive Health

Upper 3rd Floor, Royal Free Hospital

Pond Street, London NW3 2QG (UK)

E-Mail Stephen.pereira@ucl.ac.uk 
Fig. 1. Current algorithm for the management of indeterminate biliary strictures or suspected cholangiocarcinoma. Adapted from Skipworth et al. [105]. MRA = Magnetic resonance angiogram; CEA = carcinoembryonic antigen; MDT = multidisciplinary team; PVE = portal vein embolization; OLT $=$ orthotopic liver transplantation .

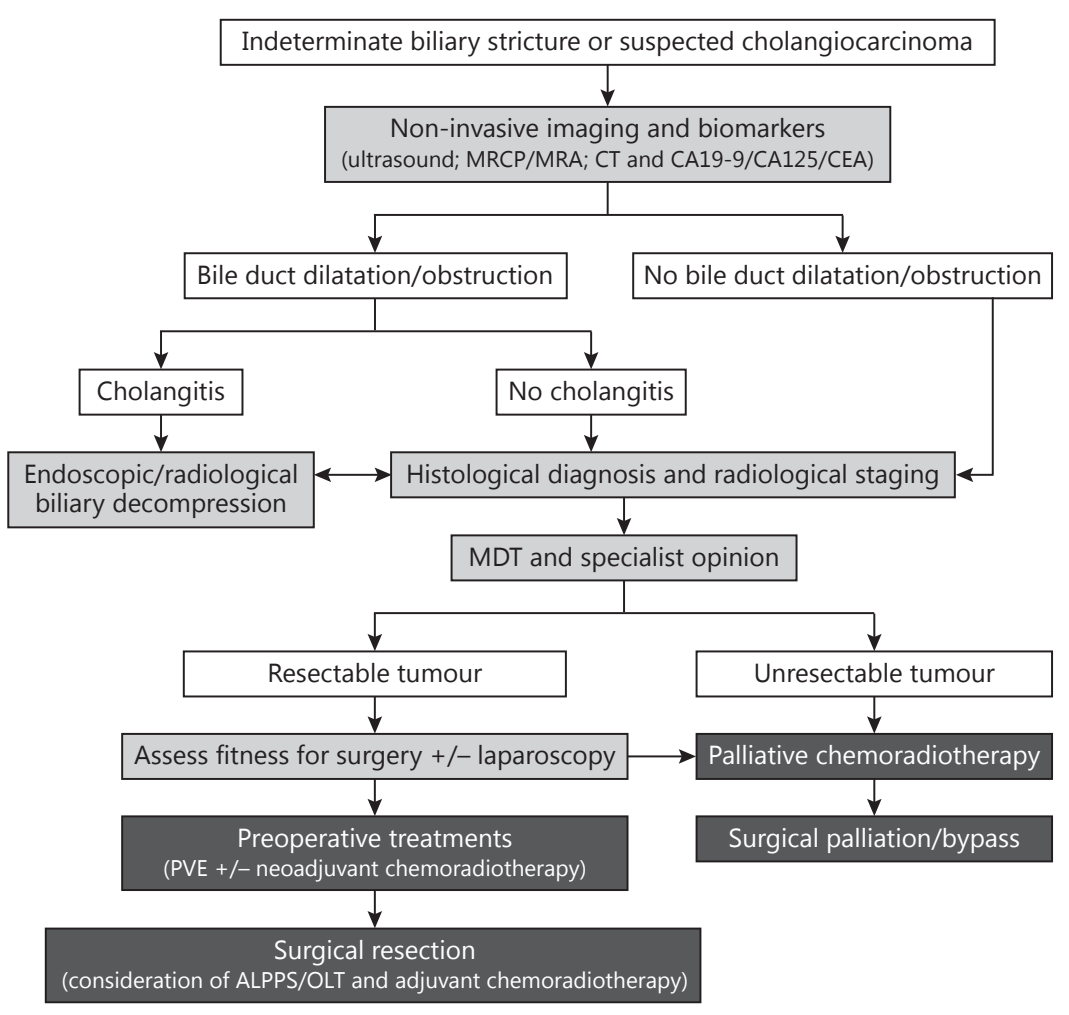

(consideration of ALPPS/OLT and adjuvant chemoradiotherapy)
The late presentation of CC patients is thought to be due in part to a lack of consistently effective biomarkers and diagnostic tools for the detection of early disease. CA (carbohydrate antigen) 19-9 remains the most widely used biomarker for the diagnosis of CC; however, it is undetectable in approximately $7 \%$ of the population who are deficient in the fucosyltransferase enzyme required for its production $[8,9]$. CA19-9 is also non-specific and can be elevated in pancreatic adenocarcinoma, primary biliary cirrhosis, cholestasis, cholangitis and in smokers [10]. However, a combination of CA19-9, magnetic resonance cholangiopancreaotography (MRCP) and endoscopic or percutaneous tissue sampling represents the standard technique for diagnosing CC. Recently, several novel endoscopic techniques such as per-oral cholangioscopy, confocal endomicroscopy and fluorescence in situ hybridisation (FISH) of biliary brushings have been shown to enhance the accuracy of standard endoscopic retrograde cholangiopancreatography (ERCP) in diagnosing CC [11-13].

Update on Cholangiocarcinoma
Prognosis in unresectable CC is poor (6-12 months) and therapeutic options have historically been limited. However, recent combinations of chemotherapy agents, biological therapies and novel local therapies have been shown to be associated with significant improvements in overall survival. Due to the rarity of the disease, many of these treatments have so far only been evaluated in small studies and validation through larger prospective studies will be required to ultimately define their role in future algorithms for the management of CC (fig. 1).

\section{Surgical Management of Resectable Cholangiocarcinoma}

\section{Preoperative Assessment and Optimisation}

Only a minority of patients with CC (15-35\%) are suitable for surgical treatment. Assessment of suitability relies on accurate cross-sectional imaging and endoscopic modalities to characterise the tumour's anatomical site as 
well as the extent of local and metastatic spread [4, 1417]. However, gradual infiltration along the biliary tract, associated with the common presence of low volume but multifocal disease, can result in CC being understaged by cross-sectional imaging. Although ultrasound and highresolution computed tomography (CT) are useful for the initial assessment of the liver and biliary system and to exclude metastatic spread, magnetic resonance imaging (MRI) with contrast angiography provides the most accurate assessment of biliary anatomy, local invasion and the extent of involvement of crucial nearby ductal and vascular structures, with a reported accuracy in localising the site and cause of biliary obstruction of 100 and 95\%, respectively [18].

The current use of positron-emission computed tomography (PET-CT) in patients with suspected CC remains limited due to lack of access and the common presence of cholangitis, which can make interpretation difficult; however, its use may become more important in the future $[19,20]$. Cytological or histological confirmation is usually obtained following cross-sectional imaging, commonly by ERCP. Endoscopic techniques remain crucial as the majority of patients diagnosed have unresectable disease and provision of adequate biliary drainage is necessary to prevent sepsis and enable palliative chemotherapy. However, when employed for diagnostic purposes, standard ERCP and brush cytology has a sensitivity for malignancy of only 9-57\% [21-24], although this may be improved by techniques such as FISH and digital image analysis, which enable the analysis of DNA abnormalities in brush cytology [23]. Further techniques, such as methylene blue, narrow-band imaging, autofluorescence, confocal laser endomicroscopy and elastic scattering spectroscopy are not routinely used but may allow an augmented view of the visualised mucosa during ERCP [25-28].

Alternative endoscopic methods include endoscopic ultrasonography with fine-needle aspiration (EUS-FNA), which provides a method for visualising and sampling the extrahepatic biliary tree, hilar masses, and perihilar ductal and vascular structures, with a sensitivity and specificity of up to 89 and $100 \%$, respectively [29], or single operator cholangioscopy systems (Spyglass; Boston Scientific Corp., Natick, Mass., USA). In cases of uncertainty regarding resectability, staging laparoscopy can provide additional information about tumour extension, liver atrophy or metastatic disease and approximately a third of such patients will be found to have irresectable disease $[17,30,31]$.

Preoperative biliary drainage in the presence of jaundice, via endoscopically-placed bile duct stents, leads to improvements in liver function and outcome following hepatic resection [32-35]. Percutaneous, transhepatic radiological techniques [e.g. percutaneous transhepatic cholangiography (PTC)] for drainage of unilateral bile ducts can also be used and may have a similar or lower complication and failure rate than endoscopic techniques [33-39].

Portal vein embolization or ligation should also be utilised preoperatively to promote hypertrophy of the liver remnant in situations where it is predicted to be less than $25 \%$ or there is unresolved preoperative jaundice, and is associated with a reported reduction in postoperative hepatic failure from 20 to $6 \%$ [40-43]. Such improvements in preoperative optimisation have led to a growing number of patients with CC being eligible for surgical resection [16].

\section{Surgical Resection}

Tumour resection with clear pathological margins offers the best long-term survival and recurrence rates in CC: an aggressive resectional strategy is therefore the mainstay of treatment $[4,15,16]$. Hepatic resection with concurrent excision and anastomosis of the portal vein and/or bile duct is considered standard treatment and has been associated with a reduction in associated morbidity and mortality [44, 45]. Specific complication, survival and recurrence rates following surgery are related to the precise nature of the surgery the patient undergoes, which in turn is dictated by the anatomical site and extent of the tumour. Patients with intrahepatic tumours will require segmental liver resection or hemihepatectomy; those with extrahepatic bile duct tumours will undergo hilar and bile duct resection, with partial hepatic resection if the bile duct bifurcation is also affected, and ampullary or distal bile duct tumours will be resected by Whipple's pancreaticoduodenectomy $[7,46]$.

Thirty-day mortality following a resection for CC ranges from 2 to $25 \%$ in various series with significant complications including bile leak, cholangitis, haemorrhage and intra-abdominal collections, occurring in up to approximately two thirds of patients $[15,17,43,47-$ 51]. Mortality rates are increased in those with significant co-morbidities, hypoalbuminaemia and jaundice in the preoperative period $[15,17,43,47-51]$ but is now similar in those undergoing extended resection (with or without vascular resection) and those having extensive hepatic resection $[49,50,52]$. The commonest postoperative complication contributing to patient death remains hepatic failure, the risk of which increases with the amount of hepatic tissue resected $[48,50]$. ALPPS 
(Associating Liver Partition with Portal vein ligation for Staged hepatectomy) is a novel two-stage resection technique involving initial in-situ transection of liver parenchyma (along the intended line of hepatic resection or falciform ligament) and portal vein ligation, with subsequent staged (7-14 days later) completion hepatectomy [53]. When compared to portal vein embolization, this strategy results in rapid volume growth of the future liver remnant (FLR; up to 75\% increase) with the most rapid growth occurring within the first 3 days of the procedure. This technique thereby enables resection of tumours that would have been previously considered to be unresectable because of the predicted small size of the FLR. ALPPS can also be utilised following initial portal vein embolization if there has been insufficient FLR volume increase $[53,54]$.

$\mathrm{CC}$ is associated with a median overall 5-year survival of 20-36 months; however, in those patients in whom complete pathological resection is possible $[16,17,55-$ 57], median survival increases to 65 months $[4,16,48$, 52]. Median survival rates also vary according to the site of the tumour (hilar tumours 18-30 months, perihilar tumours 12-24 months, extrahepatic 16-30 months) [6, $15,58-61]$ and the presence of postoperative residual disease, which leads to a significant decrease in survival rates [62].

Factors that have been associated with prolonged postoperative survival include small, early stage or well-differentiated, unifocal tumours, without regional nodal disease or macroscopic portal vein invasion $[4,15-17,49]$. However, even following successful resection, recurrence is common, occurring in up to $63 \%$ overall within 2 years [4].

\section{Liver Transplantation}

Liver transplantation negates any requirements for negative resection margins following en-bloc resection of the liver, bile ducts and hilar lymphatics, but remains limited by the scarcity of donors.

Recent results have suggested that a combination of orthotopic transplantation and neoadjuvant chemoradiotherapy regimens offer excellent long-term (5-year) survival rates of up to $82 \%$ in patients previously considered unresectable [63-65]. Recurrence rates are also lower than in resected patients (13 vs. 27\%) [63]. However, there are difficulties in direct comparison with resected patients in that transplanted patients tend to be younger, have fewer co-morbidities, have node-negative disease and almost always undergoing neoadjuvant therapy [66].

Update on Cholangiocarcinoma

\section{Treatment of Locally Advanced and Metastatic Cholangiocarcinoma}

The prognosis for patients with locally advanced or recurrent CC is poor. The goals of palliative therapy in such patients are to relieve symptoms and improve overall quality of life. There is no role for tumour debulking in advanced CC.

\section{Endoscopic Biliary Decompression in Malignant Obstruction}

Biliary drainage is essential in CC to prevent septic complications from cholangitis and enable patients to be eligible for palliative chemotherapy. Effective endoscopic biliary stenting to relieve obstruction has been associated with fewer short-term complications and longer survival (19 vs. 16.5 months), as well as being more cost-effective than surgical decompression [67]. In most centres, endoscopic or percutaneous management of biliary obstruction is therefore the standard initial approach in treating patients with malignant biliary obstruction.

\section{Distal Common Bile Duct Obstruction}

A meta-analysis of seven studies (1992-2006) compared plastic stents to self-expanding metal stents (SEMS) for malignant distal biliary obstruction and found that the relative risk of recurrent biliary obstruction was significantly lower $(\mathrm{RR}=0.52)$ in the metal stent group [68]. Since uncovered SEMS are permanent, many patients are initially managed with a plastic stent while confirmation of malignancy is obtained. However, the introduction of removable covered metal stents has changed this practice. Four randomised controlled trials have compared uncovered and covered SEMS in the management of distal biliary obstruction: in the earlier three studies, no significant differences in stent patency time, survival or complication rates were observed but covered stents did migrate more frequently [69-71]. However, in the most recent randomised trial incorporating patients with distal biliary obstruction secondary to pancreatic carcinoma, survival without stent dysfunction was significantly longer in the covered SEMS group (187 vs. 132 days, $\mathrm{p}=$ 0.043 ) and no difference in stent migration was observed [72].

\section{Proximal Biliary Obstruction}

Hilar CC can lead to disconnection of the right and left biliary systems and several studies have attempted to define optimal stenting practice in this situation. A prospective, observational study reported that plastic stent 
placement was associated with a poorer 30-day outcome (cholangitis, stent occlusion, migration, perforation, unplanned ERCP or PTC) compared with metal stents (OR 6.32; 95\% confidence interval 1.23, 32.56) [73].

A single biliary stent will achieve decompression in most patients (80\%) with hilar CC [74, 75]; however, in a retrospective study comparing the efficacy of unilateral and bilateral stent placement, functional success was significantly higher ( 97.9 vs. $84.8 \%, \mathrm{p}<0.001)$ and complications fewer ( 56.4 vs. $24.4 \%, \mathrm{p}<0.001$ ) in the bilateral SEMS group. Placement of SEMS rather than plastic stents was also associated with longer stent patency times (24 vs. 29 weeks, p < 0.0001) [76]. A further randomised study observed that rates of successful drainage were significantly higher following SEMS placement (70.4 vs. $46.3 \%, \mathrm{p}=0.1$ ) and overall survival was longer (126 vs. 49 days, $\mathrm{p}=0.002)$ [77].

\section{Novel Endoscopic and Percutaneous Local and Ablative Therapies}

Photodynamic Therapy

Photodynamic therapy (PDT) is a novel ablative technique that results in localised tissue necrosis following the application of either visible or near-infrared light (usually from a low-power, red laser) after prior administration of a photosensitising agent. Randomised studies comparing PDT with biliary stenting with stenting alone have provided conflicting results. Initial studies reported prolonged stent patency and improved survival after PDT $[78,79]$; however, a subsequent UK phase III study closed early as overall survival was longer in those treated with stenting alone [80].

Radiofrequency Ablation

Radiofrequency ablation (RFA) in combination with SEMS placement has been evaluated in a small study of 22 patients, which found that all stents were patent 30 days after the procedure [81]. Only rarely have centres described the use of RFA alone to achieve biliary drainage and randomised controlled trials are ongoing to evaluate this technique [82]. Current guidance from the National Institute for Health and Care Excellence in the UK recommends that the treatment is only carried out in specialist centres in the context of clinical trials [83].

\section{Transarterial Chemoembolization}

Transarterial delivery of embolic and cytotoxic agents leads to local tumour ischaemia and targeted chemotherapy, which, in unresectable hepatocellular carcinoma, has been associated with improved survival and resulted in its incorporation into many standard treatment algorithms [84]. Recently, it has been used in the treatment of advanced CC: a prospective study of 115 patients with unresectable CC treated with transarterial chemoembolization (TACE) found that more than half of the patients survived over 1 year (median survival 13 months) [85]. A recent retrospective study has also observed TACE to be an equivalent treatment to surgery for $\mathrm{CC}$ in those in whom an R0 resection (negative pathological margins) was not achieved [86].

\section{Chemotherapy}

A multicentre, randomised phase III study comparing cisplatin and gemcitabine to gemcitabine alone in the treatment of advanced biliary tract cancer (ABC-02) was associated with an improved median survival in the combination group (11.7 vs. 8.1) [87]. Improved progression-free survival and disease control rates were also demonstrated. Prior to the ABC-02 trial, patients with advanced CC received a range of chemotherapy regimens; however, a large retrospective review from the UK compared overall survival in patients receiving gemcitabine, fluoropyrimidine or platinum-based treatments and found that fluoropyrimidine may be as effective as gemcitabine in the treatment of advanced CC [88]. Initial prospective studies which combined gemcitabineand fluoropyrimidine-based treatments observed prolonged survival (14 months) with relatively few complications [89].

Neoadjuvant Chemoradiotherapy for CC

The primary aim of neoadjuvant therapy is to downstage malignant disease, rendering tumours suitable for surgical resection or transplantation. In other solid organ malignancies both radio- and chemotherapy have been observed to be more effective in the neoadjuvant setting. In unresectable perihilar CC, a large case series included 287 patients from 12 large-volume transplant centres in the USA. Seventy-one patients did drop out prior to liver transplantation, but recurrence-free survival rates were 78 and $65 \%$ at 2 and 5 years, respectively [90]. A further case series of 45 patients with extrahepatic disease, 12 of whom were treated neoadjuvantly. Three had a complete pathological response and 11 were able to undergo R0 resection. In addition, despite having more advanced disease at presentation, those who received neoadjuvant chemoradiotherapy had a longer 5-year survival (53 vs. $23 \%, \mathrm{p}=0.16$ ) [91]. Although these results are promising, neoadjuvant regimens are currently not part of the standard management of CC. 
Adjuvant Chemotherapy for CC

Postoperative adjuvant therapy is widely recommended for all patients with intra- or extrahepatic CC who have microscopically positive resection margins, as well as for those with a complete resection but node-positive disease. However, even following R0 resection in CC, locoregional recurrence remains high. Currently there is no established treatment protocol for those patients undergoing attempted curative resection for CC but in whom negative histological margins are not achieved. Small studies of adjuvant therapy have demonstrated promising results; the outcome of the UK BILCAP randomised controlled trial, which is evaluating the role of adjuvant chemotherapy with capecitabine (an oral 5-fluorouracil analogue), following surgical resection, is awaited.

\section{Radiotherapy}

External beam radiotherapy and intraluminal brachytherapy have been evaluated in CC [92-94]. Most studies to date have compared patients with historical controls and results have been equivocal. McMasters et al. [95] described a case series of 9 patients with extrahepatic CC who were successfully downstaged with external beam radiotherapy: $100 \%$ had negative resection margins following surgery. However, in a longer-term study by González González et al. [96], which used combinations of pre- and postoperative external beam radiotherapy, no impact on 1-, 3- or 5-year survival was observed. More recently, Gwak et al. [97] observed a downward trend in 5 -year survival following adjuvant radiotherapy (21 vs. $11.6 \%)$. Thus, large prospective studies are lacking and at present the evidence does not support the use of adjuvant radiotherapy in patients with negative resection margins [98].

Few studies have evaluated chemoradiotherapy in CC. Kim et al. [99] followed 72 extrahepatic CC patients that had undergone surgical resection (47 with negative margins and 25 with positive margins) and then went on to receive postoperative external beam radiotherapy ( $40 \mathrm{~Gy}$ ) and concomitant boluses of 5 -fluorouracil $\left(500 \mathrm{mg} / \mathrm{m}^{2}\right)$ : the 5 -year survival rates were $36 \%$ following R0 resection, $35 \%$ following R1 resection, and 0\% following R2 resection. Nakeeb and Pitt [100] similarly reported improved survival with chemoradiotherapy. Although these studies are small, they appear to support the use of radical resection followed by chemoradiotherapy in CC.

\section{Biological Therapies}

To date, several pilot studies have investigated the role of molecularly targeted therapies in CC. For example, in vitro studies of the multikinase inhibitor sorafenib have shown that it inhibits proliferation of CC cell lines in culture in a dose-dependent manner [101] and causes significant tumour suppression in a rodent model [102]. However, limited phase II studies to date in CC have not demonstrated a clinical benefit [103]. The French BINGO trial randomised 101 patients to receive gemcitabine and oxaliplatin (GEMOX) with or without cetuximab [104]. Initial findings demonstrated 4-month progression-free survival rates of $50 \%$ in the GEMOX arm and $61 \%$ in the GEMOX with cetuximab arm.

\section{Conclusion}

Despite significant advances in the treatment of patients with CC, the only potential for long-term survival remains surgical resection or liver transplantation. Unfortunately, this remains only possible in a minority of patients and both neoadjuvant and adjuvant therapies currently provide only a limited survival improvement in selected patients with advanced disease. To achieve improved outcomes, better understanding of tumour biology, combined with the development of novel diagnostic and treatment strategies, is crucial.

\section{Acknowledgements}

The work was undertaken at UCLH/UCL, which received a proportion of funding from the Department of Health's National Institute for Health Research (NIHR) Biomedical Research Centres funding scheme.

\section{Disclosure Statement}

The authors have no conflicts of interest to disclose.

\begin{tabular}{|c|c|}
\hline References & $\begin{array}{l}\text { Nakeeb A, et al: Cholangiocarcinoma. A spec- } \\
\text { trum of intrahepatic, perihilar, and distal tu- } \\
\text { mors. Ann Surg 1996;224:463-473; discus- } \\
\text { sion 473-475. } \\
2 \text { Patel T: Increasing incidence and mortality of } \\
\text { primary intrahepatic cholangiocarcinoma in } \\
\text { the United States. Hepatology 2001;33:1353- } \\
1357 \text {. } \\
3 \text { Taylor-Robinson SD, et al: Increase in mor- } \\
\text { tality rates from intrahepatic cholangiocarci- } \\
\text { noma in England and Wales 1968-1998. Gut } \\
\text { 2001;48:816-820. }\end{array}$ \\
\hline
\end{tabular}


-4 Endo I, et al: Intrahepatic cholangiocarcinoma: rising frequency, improved survival, and determinants of outcome after resection. Ann Surg 2008;248:84-96.

5 Khan SA, et al: Cholangiocarcinoma and its management. Gut 2007;56:1755-1756.

6 Khan SA, et al: Guidelines for the diagnosis and treatment of cholangiocarcinoma: consensus document. Gut 2002;51(suppl 6):VI1VI9.

7 Khan SA, et al: Changing internationals trends in mortality rates for liver, biliary and pancreatic tumours. J Hepatol 2002;37:806813.

8 Nehls O, Gregor M, Klump B: Serum and bile markers for cholangiocarcinoma. Semin Liver Dis 2004;24:139-154.

9 Locker GY, et al: ASCO 2006 update of recommendations for the use of tumor markers in gastrointestinal cancer. J Clin Oncol 2006; 24:5313-5327.

10 Bonney GK, et al: Circulating markers of biliary malignancy: opportunities in proteomics? Lancet Oncol 2008;9:149-158.

-11 Meining A, et al: Direct visualization of indeterminate pancreaticobiliary strictures with probe-based confocal laser endomicroscopy: a multicentre experience. Gastrointest Endosc 2011;74:961-968.

12 Tischendorf JJ, et al: Cholangioscopic characterization of dominant bile duct stenoses in patients with primary sclerosing cholangitis. Endoscopy 2006;38:665-669.

13 Moreno Luna LE, et al.: Advanced cytologic techniques for the detection of malignant pancreobiliary strictures. Gastroenterology 2006;131:1064-1072.

14 Bismuth H, Nakache R, Diamond T: Management strategies in resection for hilar cholangiocarcinoma. Ann Surg 1992;215:31-38.

$\checkmark 15$ Witzigmann $\mathrm{H}$, et al: Surgical and palliative management and outcome in 184 patients with hilar cholangiocarcinoma: palliative photodynamic therapy plus stenting is comparable to R1/R2 resection. Ann Surg 2006; 244:230-239.

16 Ito F, et al: Resection of hilar cholangiocarcinoma: concomitant liver resection decreases hepatic recurrence. Ann Surg 2008;248:273279.

17 Jarnagin WR, et al: Staging, resectability, and outcome in 225 patients with hilar cholangiocarcinoma. Ann Surg 2001;234:507-517; discussion 517-519.

18 Romagnuolo J, et al: Magnetic resonance cholangiopancreatography: a meta-analysis of test performance in suspected biliary disease. Ann Intern Med 2003;139:547-557.

19 Corvera CU, et al: ${ }^{18} \mathrm{~F}$-fluorodeoxyglucose positron emission tomography influences management decisions in patients with biliary cancer. J Am Coll Surg 2008;206:57-65.
20 Furukawa H, et al: Prognostic importance of standardized uptake value on ${ }^{18} \mathrm{~F}$-fluorodeoxyglucose-positron emission tomography in biliary tract carcinoma. J Surg Oncol 2009; 100:494-499.

21 Baron TH, et al: A prospective comparison of digital image analysis and routine cytology for the identification of malignancy in biliary tract strictures. Clin Gastroenterol Hepatol 2004;2:214-219.

22 De Bellis M, et al: Tissue sampling at ERCP in suspected malignant biliary strictures (Part 1). Gastrointest Endosc 2002;56:552-561.

23 Moreno Luna LE, et al: Advanced cytologic techniques for the detection of malignant pancreatobiliary strictures. Gastroenterology 2006;131:1064-1072.

24 Harewood GC, et al: Prospective, blinded assessment of factors influencing the accuracy of biliary cytology interpretation. Am J Gastroenterol 2004;99:1464-1469.

25 Meining A, et al: Detection of cholangiocarcinoma in vivo using miniprobe-based confocal fluorescence microscopy. Clin Gastroenterol Hepatol 2008;6:1057-1060.

26 Huggett MT, et al: Photodynamic therapy of pancreatic cancer and elastic scattering spectroscopy of the duodenal mucosa for the detection of pancreaticobiliary malignancy. Proc SPIE7886, Optical Methods for Tumor Treatment and Detection: Mechanisms and Techniques in Photodynamic Therapy, 2011. $\mathrm{XX}, 78860 \mathrm{~J}$

27 Hoffman A, et al: Methylene blue-aided cholangioscopy in patients with biliary strictures: feasibility and outcome analysis. Endoscopy 2008;40:563-571.

28 Lu XL, Itoi T, Kubota K: Cholangioscopy by using narrow-band imaging and transpapillary radiotherapy for mucin-producing bile duct tumour. Clin Gastroenterol Hepatol 2009;7:e34-e35.

29 Fritscher-Ravens A, et al: EUS-guided fineneedle aspiration of suspected hilar cholangiocarcinoma in potentially operable patients with negative brush cytology. Am J Gastroenterol 2004;99:45-51.

30 Goere D, et al: Utility of staging laparoscopy in subsets of biliary cancers: laparoscopy is a powerful diagnostic tool in patients with intrahepatic and gallbladder carcinoma. Surg Endosc 2006;20:721-725.

31 Corvera CU, Weber SM, Jarnagin WR: Role of laparoscopy in the evaluation of biliary tract cancer. Surg Oncol Clin N Am 2002;11: 877-891.

32 Grandadam S, et al: Role of preoperative optimization of the liver for resection in patients with hilar cholangiocarcinoma type III. Ann Surg Oncol 2010;17:3155-3161.

33 Pitt HA, et al: Does preoperative percutaneous biliary drainage reduce operative risk or increase hospital cost? Ann Surg 1985;201: 545-553.
34 Hatfield AR, et al: Preoperative external biliary drainage in obstructive jaundice. A prospective controlled clinical trial. Lancet 1982; 2:896-899.

35 Blamey SL, et al: Prediction of risk in biliary surgery. Br J Surg 1983;70:535-538.

36 Hochwald SN, et al: Association of preoperative biliary stenting with increased postoperative infectious complications in proximal cholangiocarcinoma. Arch Surg 1999; 134: 261-266.

37 Mansfield SD, et al: Management of hilar cholangiocarcinoma in the North of England: pathology, treatment, and outcome. World J Gastroenterol 2005;11:7625-7630.

-38 Cortes A, et al: Effect of bile contamination on immediate outcomes after pancreaticoduodenectomy for tumor. J Am Coll Surg 2006;202: 93-99.

39 Nagino M, et al: Preoperative biliary drainage for biliary tract and ampullary carcinomas. J Hepatobiliary Pancreat Surg 2008;15:25-30.

40 Nimura Y, et al: Aggressive preoperative management and extended surgery for hilar cholangiocarcinoma: Nagoya experience. J Hepatobiliary Pancreat Surg 2000;7:155-162.

-41 Hemming AW, et al: Preoperative portal vein embolization for extended hepatectomy. Ann Surg 2003;237:686-691; discussion 691-693.

42 Abdalla EK, et al: Extended hepatectomy in patients with hepatobiliary malignancies with and without preoperative portal vein embolization. Arch Surg 2002;137:675-680; discussion 680-681.

43 Igami T, et al: Surgical treatment of hilar cholangiocarcinoma in the 'new era': the Nagoya University experience. J Hepatobiliary Pancreat Surg 2010;17:449-454.

44 Shaib Y, El-Serag HB: The epidemiology of cholangiocarcinoma. Semin Liver Dis 2004; 24:115-125.

-45 Khan SA, et al: Changing international trends in mortality rates for liver, biliary and pancreatic tumours. J Hepatol 2002;37:806-813.

46 Jarnagin WR, Shoup M: Surgical management of cholangiocarcinoma. Semin Liver Dis 2004;24:189-199.

-47 Van den Broek MA, et al: Feasibility of randomized controlled trials in liver surgery using surgery-related mortality or morbidity as endpoint. Br J Surg 2009;96:1005-1014.

48 Gerhards MF, et al: Evaluation of morbidity and mortality after resection for hilar cholangiocarcinoma - a single-center experience. Surgery 2000;127:395-404.

49 Ebata T, et al: Hepatectomy with portal vein resection for hilar cholangiocarcinoma: audit of 52 consecutive cases. Ann Surg 2003;238: 720-727.

50 Neuhaus P, et al: Surgical management of proximal bile duct cancer: extended right lobe resection increases resectability and radicality. Langenbecks Arch Surg 2003;388:194200 . 
$51 \mathrm{Su} \mathrm{CH}$, et al: Factors influencing postoperative morbidity, mortality, and survival after resection for hilar cholangiocarcinoma. Ann Surg 1996;223:384-394.

52 Kondo S, et al: Forty consecutive resections of hilar cholangiocarcinoma with no postoperative mortality and no positive ductal margins: results of a prospective study. Ann Surg 2004; 240:95-101.

53 Schnitzbauer AA, et al: Right portal vein ligation combined with in situ splitting induces rapid left lateral liver lobe hypertrophy enabling two-staged extended right hepatic resection in small-for-size settings. Ann Surg 2012;255:405-414.

-54 Knoefel WT, et al: In situ liver transection with portal vein ligation for rapid growth of the future liver remnant in two-stage liver resection. Br J Surg 2013;100:388-394.

55 Nishio H, Nagino M, Nimura Y: Surgical management of hilar cholangiocarcinoma: the Nagoya experience. HPB (Oxford) 2005;7: 259-262.

56 Hidalgo E, et al: Surgery for hilar cholangiocarcinoma: the Leeds experience. Eur J Surg Oncol 2008;34:787-794.

57 Silva MA, et al: Surgery for hilar cholangiocarcinoma; a 10-year experience of a tertiary referral centre in the UK. Eur J Surg Oncol 2005;31:533-539.

58 Reding R, et al: Surgical management of 552 carcinomas of the extrahepatic bile ducts (gallbladder and periampullary tumors excluded). Results of the French Surgical Association Survey. Ann Surg 1991;213:236-241.

59 Aljiffry M, et al: Evidence-based approach to cholangiocarcinoma: a systematic review of the current literature. J Am Coll Surg 2009; 208:134-147.

60 Henson DE, Albores-Saavedra J, Corle D: Carcinoma of the extrahepatic bile ducts. Histologic types, stage of disease, grade, and survival rates. Cancer 1992;70:1498-1501.

61 Iwatsuki S, et al: Treatment of hilar cholangiocarcinoma (Klatskin tumors) with hepatic resection or transplantation. J Am Coll Surg 1998; 187:358-364.

62 Witzigmann $\mathrm{H}$, et al: Surgical and palliative management and outcome in 184 patients with hilar cholangiocarcinoma: palliative photodynamic therapy plus stenting is comparable to R1/R2 resection. Ann Surg 2006; 244:230-239.

63 Rea DJ, et al: Liver transplantation with neoadjuvant chemoradiation is more effective than resection for hilar cholangiocarcinoma. Ann Surg 2005;242:451-458; discussion 458461.

64 Rea DJ, et al: Transplantation for cholangiocarcinoma: when and for whom? Surg Oncol Clin N Am 2009; 18:325-337, ix.

65 Heimbach JK, et al: Liver transplantation for unresectable perihilar cholangiocarcinoma. Semin Liver Dis 2004;24:201-207.
66 Callery MP: Transplantation for cholangiocarcinoma: advance or supply-demand dilemma? Gastroenterology 2006; 130:22422244; discussion 2244

67 Martin RC 2nd, et al: Cost comparison of endoscopic stenting versus surgical treatment for unresectable cholangiocarcinoma. Surg Endosc 2002;16:667-670.

68 Moss AC, Morris E, Leyden J, MacMathuna P: Malignant distal biliary obstruction: a systemic review and meta-analysis of endoscopic and surgical bypass results. Cancer Treat Rev 2007;33:213-221.

69 Krokidis M, et al: Percutaneous treatment of malignant jaundice due to extrahepatic cholangiocarcinoma: covered Viabil stent versus uncovered wallstents. Cardiovasc Intervent Radiol 2010;33:97-106.

70 Isayama $\mathrm{H}$, et al: A prospective randomised study of 'covered' versus 'uncovered' diamond stents for the management of distal malignant biliary obstruction. Gut 2004;53:729734.

71 Kullman E, et al: Covered versus uncovered self-expandable nitinol stents in the palliative treatment of malignant distal biliary obstruction: results from a randomized, multicenter study. Gastrointest Endosc 2010;72: 915-923.

72 Kitano M, et al: Covered self-expanding metal stents with anti-migration system improve patency duration without increased complications compared with uncovered stents for distal biliary obstruction caused by pancreatic carcinoma: a randomized multicenter trial. Am J Gastroenterol 2013;305:1-10.

73 Perdue DG, et al: Plastic versus self-expanding metallic stents for malignant hilar biliary obstruction: a prospective multicentre observational cohort study. J Clin Gastroenterol 2008;42:1040-1046.

74 Hintze RE, et al: Magnetic resonance cholangiopancreatography-guided unilateral endoscopic stent placement for Klatskin tumors. Gastrointest Endosc 2001;53:40-46.

75 Freeman ML, Overby C: Selective MRCP and CT-targeted drainage of malignant hilar biliary obstruction with self-expanding metallic stents. Gastrointest Endosc 2003;58:41-49.

76 Liberato MJ, Canena J: Endoscopic stenting for hilar cholangiocarcinoma: efficacy of unilateral and bilateral placement of plastic and metal stents in a retrospective review of 480 patients. BMC Gastroenterol 2012;12:103.

77 Sangchan A, et al: Efficacy of metal and plastic stents in unresectable complex hilar cholangiocarcinoma: a randomized controlled trial. Gastrointest Endosc 2012;76:93-99.

78 Zoepf T, et al: Palliation of nonresectable bile duct cancer: improved survival after photodynamic therapy. Am J Gastroenterol 2005;100: 2426-2430.

79 Gerhardt T, et al: Combination of bilateral metal stenting and trans-stent photodynamic therapy for palliative treatment of hilar cholangiocarcinoma. Z Gastroenterol 2010;48: 28-32.
80 Pereira SP, et al: Photostent-02: porfimer sodium photodynamic therapy plus stenting alone in patients with advanced or metastatic cholangiocarcinomas and other biliary tract tumours: a multicentre, randomised phase III trial. 2010 European Society for Medical Oncology Congress, Milan, October 8-12, 2010, abstract $802 \mathrm{O}$.

81 Steel AW, et al: Endoscopically applied radiofrequency ablation appears to be safe in the treatment of malignant biliary obstruction. Gastrointest Endosc 2011;73:149-153.

82 Shariff MI, Khan S, Westaby D: The palliation of cholangiocarcinoma. Curr Opin Support Palliat Care 2013;7:168-174.

83 NICE: Using radiofrequency energy to treat malignant bile or pancreatic duct obstructions caused by cholangiocarcinoma or pancreatic adenocarcinoma. 2013.

84 Llovet JM, Brú C, Bruix J: Prognosis of hepatocellular carcinoma: the BCLC staging classification. Semin Liver Dis 1999;19:329-338.

85 Vogl TJ, et al: Transarterial chemoembolization in the treatment of patients with unresectable cholangiocarcinoma: results and prognostic factors governing treatment success. Int J Cancer 2011;131:733-740.

86 Scheuermann U, et al: Comparison of resection and transarterial chemoembolisation in the treatment of advanced intrahepatic cholangiocarcinoma - a single-center experience. Eur J Surg Oncol 2013;39:593-600.

87 Valle J, et al: Cisplatin plus gemcitabine versus gemcitabine for biliary tract cancer. N Engl J Med 2010;362:1273-1281.

88 Huggett MT, et al: Outcome and patterns of care in advanced biliary tract carcinoma (ABC): experience from two tertiary institutions in the United Kingdom. Tumori 2014 (in press).

89 Knox JJ, et al: Combining gemcitabine and capecitabine in patients with advanced biliary cancer: a phase II trial. J Clin Oncol 2005;23: 2332-2338.

90 Darwish Murad S, et al: Efficacy of neoadjuvant chemoradiation, followed by liver transplantation, for perihilar cholangiocarcinoma at 12 US centers. Gastroenterology 2012;143: 88-98.

91 Nelson JW, et al: Concurrent chemoradiotherapy in resected extrahepatic cholangiocarcinoma. Int J Radiat Oncol Biol Phys 2009; 73:148-153.

92 Saito H, et al: Radiation therapy and photodynamic therapy for biliary tract and ampullary carcinomas. J Hepatobiliary Pancreat Surg 2008;15:63-68.

93 Kuvshinoff BW, et al: Palliation of irresectable hilar cholangiocarcinoma with biliary drainage and radiotherapy. Br J Surg 1995;82: $1522-1525$.

94 Fletcher MS, et al: Treatment of hilar carcinoma by bile drainage combined with internal radiotherapy using iridium-192 wire. $\mathrm{Br} \mathrm{J}$ Surg 1983;70:733-735. 
95 McMasters KM, et al: Neoadjuvant chemoradiation for extrahepatic cholangiocarcinoma. Am J Surg 1997; 174:605-608; discussion 608609.

96 González González D, et al: Role of radiotherapy, in particular intraluminal brachytherapy, in the treatment of proximal bile duct carcinoma. Ann Oncol 1999;10(suppl 4):215-220.

97 Gwak HK, Kim WC, Kim HJ, Park JH: Extrahepatic bile duct cancers: surgery alone versus surgery plus postoperative radiation therapy. Int J Radiat Oncol Biol Phys 2010;78:194-198.

98 Pitt HA, et al: Perihilar cholangiocarcinoma. Postoperative radiotherapy does not improve survival. Ann Surg 1995;221:788-797; discussion 797-798.
99 Kim S, et al: Role of postoperative radiotherapy in the management of extrahepatic bile duct cancer. Int J Radiat Oncol Biol Phys 2002;54:414-419.

100 Nakeeb A, Pitt HA: Radiation therapy, chemotherapy and chemoradiation in hilar cholangiocarcinoma. HPB (Oxford) 2005;7: 278-282.

101 Huether A, et al: Sorafenib alone or as combination therapy for growth control of cholangiocarcinoma. Biochem Pharmacol 2007; 73:1308-1317.
102 Blechacz BR, Sanchez W, Gores GJ: A conceptual proposal for staging ductal cholangiocarcinoma. Curr Opin Gastroenterol 2009;25:238-239.

103 Bengala C, et al: Sorafenib in patients with advanced biliary tract carcinoma: a phase II trial. Br J Cancer 2010;102:68-72.

104 Tournigand C, Malka D, Desrame J: 45th Congress of the American Society of Clinical Oncology (ASCO), Orlando, May 30-June 2 2009. J Chir (Paris) 2009;146:311-315.

105 Skipworth JR, et al: Review article: surgical, neo-adjuvant and adjuvant management strategies in biliary tract cancer. Aliment Pharmacol Ther 2011;34:1063-1078. 